\title{
Article
}

\section{Cross-National Study of Worrying, Loneliness, and Mental Health during the COVID-19 Pandemic: A Comparison between Individuals with and without Infection in the Family}

\author{
Tore Bonsaksen $1,2, * \mathbb{C}$, Janni Leung ${ }^{3} \mathbb{D}$, Mariyana Schoultz ${ }^{4} \mathbb{D}$, Hilde Thygesen ${ }^{5,6}$, Daicia Price ${ }^{7} \mathbb{D}$, \\ Mary Ruffolo ${ }^{7(-)}$ and Amy Østertun Geirdal ${ }^{8}$ (i)
}

Citation: Bonsaksen, T.; Leung, J.; Schoultz, M.; Thygesen, H.; Price, D.; Ruffolo, M.; Geirdal, A.Ø. Cross-National Study of Worrying, Loneliness, and Mental Health during the COVID-19 Pandemic: A Comparison between Individuals with and without Infection in the Family. Healthcare 2021, 9, 903. https://doi.org/10.3390/ healthcare 9070903

Academic Editor: Alyx Taylor

Received: 7 June 2021

Accepted: 13 July 2021

Published: 16 July 2021

Publisher's Note: MDPI stays neutral with regard to jurisdictional claims in published maps and institutional affiliations.

Copyright: (c) 2021 by the authors. Licensee MDPI, Basel, Switzerland. This article is an open access article distributed under the terms and conditions of the Creative Commons Attribution (CC BY) license (https:// creativecommons.org/licenses/by/ $4.0 /)$.
1 Department of Health and Nursing Sciences, Faculty of Social and Health Sciences, Inland University of Applied Sciences, Hamarvegen 112, 2418 Elverum, Norway

2 Faculty of Health Studies, VID Specialized University, 4306 Sandnes, Norway

3 Faculty of Health and Behavioural Science, The University of Queensland, St. Lucia, QLD 4072, Australia; j.leung1@uq.edu.au

4 Faculty of Health and Life Sciences, Northumbria University, Newcastle upon Tyne NE1 8ST, UK; mariyana.schoultz@northumbria.ac.uk

5 Department of Occupational Therapy, Prosthetics and Orthotics, Faculty of Health Sciences, Oslo Metropolitan University, 0130 Oslo, Norway; hilde.thygesen@oslomet.no

6 Faculty of Health Studies, VID Specialized University, 0370 Oslo, Norway

7 School of Social Work, University of Michigan, Ann Arbor, MI 48109, USA; daiciars@umich.edu (D.P.); mruffolo@umich.edu (M.R.)

8 Department of Social Work, Faculty of Social Sciences, Oslo Metropolitan University, 0130 Oslo, Norway; amyoge@oslomet.no

* Correspondence: tore.bonsaksen@inn.no

\begin{abstract}
Objective: The objective of this study was to examine differences in worry, loneliness, and mental health between those individuals infected by COVID-19 or having someone their family infected, and the rest of the population. Methods: A cross-sectional online survey was conducted in Norway, UK, USA, and Australia during April/May 2020. Participants $(n=3810)$ were recruited via social media postings by the researchers and the involved universities. Differences between those with and without infection in the family were investigated with chi-square tests and independent $t$-tests. Multiple regression analyses were used to assess associations between sociodemographic variables and psychological outcomes (worry, loneliness, and mental health) in both groups. Results: Compared to their counterparts, participants with infection in the family reported higher levels of worries about themselves $(p<0.05)$ and their family members $(p<0.001)$ and had poorer mental health $(p<0.05)$. However, the effect sizes related to the differences were small. The largest effect $(d=0.24)$ concerned worries about their immediate family. Poorer psychological outcomes were observed in those who were younger, female, unemployed, living alone and had lower levels of education, yet with small effect sizes. Conclusions: In view of the small differences between those with and without infection, we generally conclude that the mental health effects of the COVID-19 situation are not limited to those who have been infected or have had an infection within the family but extend to the wider population.
\end{abstract}

Keywords: COVID-19; coronavirus; cross-national study; loneliness; mental health; pandemic; social distancing; worry

\section{Introduction}

As the COVID-19 pandemic became a reality in Western countries in the beginning of March 2020, strict national policies regarding public behavior were implemented in countries throughout the world, including Europe, America, and Australia. In conjunction with hygiene rules, social distancing became the main policy for public behavior $[1,2]$. 
The policy implied having minimal contact with persons from outside the household, and people were generally encouraged to stay at home. Nurseries, schools, and universities were closed, and practically overnight, working from home and attending online classes became the new standard for workers and students. Flights and travels were cancelled, as were all sports, religious, and cultural events. Non-vital businesses requiring physical proximity, such as hairdressers and physiotherapy clinics, were closed. Consequently, many businesses experienced financial problems, and many employees were temporarily furloughed from their jobs [3]. As higher age and chronic disease have been associated with increased risk of experiencing severe outcomes from contracting COVID-19 infection [4], individuals in these groups were instructed to be particularly careful regarding exposure to other people.

While studies of medical treatments, drugs and vaccines were developed rapidly after the pandemic outbreak, studies concerned with the psychological and social consequences of the global response to the pandemic are of no less importance. Social distancing, especially the more severe forms such as social isolation and quarantine without the possibility of meeting other people, can result in high levels of psychological distress and even post-traumatic stress disorder $[5,6]$. Social isolation has also been associated with increased risks of chronic disease and premature mortality [7]. In a study conducted during the first phase of the COVID-19 outbreak in China, more than half of the respondents rated the psychological impact as moderate-to-severe, and about one-third reported moderate-tosevere anxiety [8]. However, access to specific and up to date health information and using precautionary measures were associated with lower levels of stress, anxiety, and depression.

From a global perspective, isolation and social distancing have been included among a range of factors that may aggravate the longer-term impact of COVID-19 on mental health $[9,10]$. On the other hand, previous research has demonstrated that adequate disease knowledge (health literacy) and social contact and support are strong predictors of health outcomes [11,12]. Social contact and support serve as a 'buffer' between stress and mental health problems [13]. This is not only when support is received but also when support is given - the findings from a Japanese study showed that men under stressful circumstances were less depressed when they received or provided social support [14]. Thus, the social distancing measures taken to avoid the spread of COVID-19 appear to have the potential to undermine people's mental health and sense of belonging in the community.

Physical diseases have frequently been shown to be associated with poorer mental health, among young [15] and older people alike [16]. Thus, individuals infected with COVID-19 may also experience reduced mental health, although with substantial individual variation. However, in view of the everyday life constraints that people have experienced during a time of social distancing, mental health may not only be reduced among those who have been infected by the disease or have witnessed family members or close ones fall ill. The hypothesis driving this study is that the social distancing context of COVID-19 affects the public mental health, and not only the mental health of those who have been directly exposed to the disease. To that effect, the social media coverage of the COVID-19 pandemic, often showcasing the worst possible effects of the disease (e.g., people receiving intensive care in hospitals, alarming death rates), has been enormous. A recent study from China found that participants with more exposure to social media had higher odds of anxiety, alone and in combination with depression [17]. General population studies comparing mental health measures of those directly affected by COVID-19 with those who were not, are lacking. This study contributes to filling this gap in the literature. If the study hypothesis is supported, mental health policies in the pandemic context would need to target the general population, and not only those who have been most directly affected. Therefore, the aim of this study was to examine the differences in worry, loneliness, and mental health between those individuals infected by COVID-19 or having someone their family infected, and the rest of the population. The specific research question for the study was: Are people who have been infected with the coronavirus, or have had 
family members infected, more worried, lonely, and mentally distressed compared to those without this experience with COVID-19 infection?

\section{Methods}

\subsection{Setting}

The researchers behind the study were based in four different countries: Norway, USA, UK, and Australia. Therefore, the general population in these countries were invited in April/May 2020 to participate in a self-administered survey that was distributed via different social media, such as Facebook, Instagram, and Twitter. Each participating country had a landing site for the survey at the involved universities. These were Oslo Metropolitan University, Norway; University of Michigan, USA; University of Salford, UK; and University of Queensland, Australia. A.Ø.G. from Oslo Metropolitan University initiated the overall project, but each of the universities had a project lead. The survey was translated from Norwegian to English by the researchers according to the language and cultural context where the survey was to be used. All data used in the study are based on responses to the online survey.

\subsection{Participants}

A convenience sample was recruited through the use of various social media linking to the online survey (outlined above). To be included in the study, participants were required to be 18 years or older; to understand the language in which the survey was presented (Norwegian or English), and to be living in one of the relevant countries at the time of the survey (Norway, USA, UK or Australia).

\subsection{Measures}

\subsubsection{Sociodemographic Characteristics}

Sociodemographic variables included age group (18-29 years, 30-39 years, 40-49 years, 50-59 years, 60-69 years, 70 years and above), gender (male, female, other/not stated), living area (rural/farming area, small town, medium-sized city, large city), highest completed education level (elementary school, high school, associated/technical degree, bachelor's degree, master's/doctoral degree), cohabitation (living with spouse or partner), and employment status (having full-time or part-time employment versus not having employment).

\subsubsection{Worry}

Three aspects of worry were measured. The participants were asked: (i) to what extent are you worried about your own situation, (ii) to what extent are you worried about your immediate family, and (iii) to what extent are you worried about the future? All items had the following response options: (1) not at all, (2) slightly worried, (3) worried, (4) very worried, and (5) overwhelmed. The items were constructed for this study and analysed as individual items.

\subsubsection{Loneliness}

Loneliness was measured with the Loneliness Scale [18] which consists of six statements, all of which rated from 0 (totally disagree) to 4 (totally agree). This scale was designed to measure two different aspects of loneliness, "emotional loneliness" and "social loneliness". Previous factor-analytic studies have found the six statements to load on two different factors, and that they, therefore, should be treated as constituting two different scales reflecting the two different aspects of loneliness [18,19]. Internal consistencies (Cronbach's $\alpha$ ) in this study were 0.66 and 0.86 for the emotional loneliness and social loneliness scales, respectively.

\subsubsection{Mental Health}

General Health Questionnaire 12 (GHQ-12) is widely used as a self-report measure of mental health $[20,21]$. A large number of studies in the general adult, clinical, work 
and student population have provided support for its validity across samples and contexts [22-26]. Six items of the GHQ-12 are phrased positively (e.g., 'able to enjoy day-to-day activities'), while six items are phrased as a negative experience (e.g., 'felt constantly under strain'). For each item, the person indicates the degree to which the item content has been experienced during the two preceding weeks, using four response categories ('less than usual', 'as usual', 'more than usual' or 'much more than usual'). Items are scored between 0 and 3 , and positively formulated items are recoded prior to analysis. As a result, the GHQ-12 scale score range is $0-36$, with higher scores indicating poorer mental health (more psychological distress). Cronbach's $\alpha$ for the GHQ-12 was 0.87 .

\subsubsection{COVID-19 Infection}

Participants were asked two questions relating to COVID-19 infection: (i) have you been infected by COVID-19; and (ii) has someone in your immediate family been infected by COVID-19? Both questions were answered with 'yes', 'no' or 'don't know'. In view of the small number of participants who reported COVID-19 infection, we compared those who had been personally infected with COVID-19 $(n=52,1.4 \%)$ with those reporting that someone in the immediate family had been infected $(n=373,9.8 \%)$. Between these groups, the difference regarding social loneliness $(M=4.9$ versus $M=3.9, p<0.05)$ was the only difference reaching statistical significance. Therefore, we grouped those individuals personally infected with individuals who had a family member infected and with those reporting both, into one category $(n=504,13.2 \%)$. The rest of the participants $(n=3306$, $86.8 \%$ ) constituted the comparison group (those indicating not known infection or no infection, either personally or within the family). For the remainder of this article, we will label those participants with infection personally or within the immediate family as 'with infection', while the rest of the participants will be labelled 'without infection' in the family.

\subsection{Statistical Analysis}

The overall sample, and each of the national subsamples, were described with frequencies and percentages for categorical variables and means and standard deviations for continuous variables. Overall differences in proportions between groups were analyzed with the chi-square test.

Methodology studies have found that deviations from the normal distribution is common and often unproblematic in large public health datasets [27], and that parametric statistical tests can be used in large studies instead of non-parametric tests, even in cases of heavily skewed distributions [27], given that conditions for their use are met. In our study, the outcome variables' distribution deviated from the normal distribution (all KolmogorovSmirnov tests $p<0.001$ ) but was not heavily skewed (skewness $<0.80$ for all). When comparing the groups using both parametric and non-parametric methods, the results were identical across methods. Thus, we proceeded with the use of parametric statistical tests. Differences in worry, loneliness, and mental health between those with and without infection were analyzed with independent $t$-tests for the whole sample and for each of the four countries. A series of linear regression analyses, stratified on infection status, was used to assess associations between sociodemographic variables and a range of outcomes: worry for oneself, worry for family members, worry about the future, emotional loneliness, social loneliness, and mental health. In each of the regression analyses, included independent variables were age group, gender, education level, employment and living with spouse or partner. Statistical significance was set at $p<0.05$. Missing values were managed with casewise deletion, resulting in $n$ varying between analyses.

\subsection{Ethics}

The data in this cross-sectional cross-national study were collected anonymously. The study was quality assured and approved by Oslo Metropolitan University and by the Regional Committee for medical and health research ethics (REK; project reference 132066) in Norway. In the USA, it was reviewed by the University of Michigan Institutional Review 
Board for Health Sciences and Behavioral Sciences (IRB HSBS) and designated as exempt (HUM00180296). Similarly, it was reviewed by the University Health Research Ethics (HSR1920-080) in the UK and the University of Queensland Human Research Ethics Office (HSR1920-080; 2020000956) in Australia.

\section{Results}

\subsection{Participants}

The sociodemographic characteristics of the sample subgroups are displayed in Table 1. Overall, the distribution of sociodemographic characteristics was similar between those with and without infection. Fifty-nine percent of the sample was below the age of 50 years, and $74 \%$ had a bachelor's degree education or higher. Seventy-one percent were in employment, and $85 \%$ reported living with a spouse or partner. There was a larger proportion of women who were classified as 'with infection', compared to men.

Table 1. Sociodemographic characteristics among individuals with and without COVID-19 infection.

\begin{tabular}{|c|c|c|c|c|c|}
\hline \multirow[b]{2}{*}{ Characteristics } & \multicolumn{2}{|c|}{$\begin{array}{l}\text { Without Infection } \\
(n=3306,86.8 \%)\end{array}$} & \multicolumn{2}{|c|}{$\begin{array}{l}\text { With Infection } \\
(n=504,13.2 \%)\end{array}$} & \multirow[b]{2}{*}{$p$} \\
\hline & $n$ & $\%$ & $n$ & $\%$ & \\
\hline Age group & & & & & 0.56 \\
\hline $18-29$ years & 619 & 87.8 & 86 & 12.2 & \\
\hline 30-39 years & 628 & 88.1 & 85 & 11.9 & \\
\hline 40-49 years & 721 & 87.2 & 106 & 12.2 & \\
\hline 50-59 years & 617 & 85.3 & 106 & 14.7 & \\
\hline 60-69 years & 526 & 85.9 & 86 & 14.1 & \\
\hline $70+$ years & 191 & 85.3 & 33 & 14.7 & \\
\hline Gender & & & & & $<0.01$ \\
\hline Male & 647 & 90.1 & 71 & 9.9 & \\
\hline Female & 2607 & 85.9 & 427 & 14.1 & \\
\hline Living area & & & & & 0.35 \\
\hline Rural/farming & 244 & 86.5 & 38 & 13.5 & \\
\hline Small town & 736 & 87.3 & 107 & 12.7 & \\
\hline Medium-sized city & 1079 & 87.9 & 149 & 12.1 & \\
\hline Large city & 1247 & 85.6 & 210 & 14.4 & \\
\hline Education level & & & & & 0.27 \\
\hline Lower education & 848 & 85.7 & 141 & 14.3 & \\
\hline Bachelor's degree or higher education & 2457 & 87.1 & 363 & 12.9 & \\
\hline Living with spouse/partner & & & & & 0.93 \\
\hline Yes & 2020 & 86.4 & 319 & 13.6 & \\
\hline No & 1041 & 86.5 & 163 & 13.5 & \\
\hline Employment & & & & & \\
\hline Yes, full-time or part-time & 2340 & 86.9 & 352 & 13.1 & 0.72 \\
\hline No & 966 & 86.5 & 151 & 13.5 & \\
\hline
\end{tabular}

Note. Statistical tests are chi-square tests. Proportions are within categories.

\subsection{Differences between Participants with and without Infection}

Table 2 displays the results from the independent $t$-tests of differences in the whole sample between those with and without infection. Compared to their counterparts, participants with infection in the family rated that they were significantly more worried about their own situation and about family members, and they had poorer mental health. However, the effect sizes related to the differences were small, with the largest effect $(d=0.24)$ concerned with the difference in worry about the immediate family.

The proportion of participants exposed to infection differed between countries $(p<0.001)$. Norway had 124 individuals with infection (16.1\% of the 771 participants from Norway), while the UK had 224 (16.3\% of the participants from the UK), USA had 140 (10.1\% of the participants from USA) and Australia had 16 (5.9\% of the participants from Australia). Rerunning the analyses by country, the main pattern of small to negligible differences was 
retained, with some minor divergence from the overall results. In Norway, participants with infection (self or within the immediate family) were more worried about their family members $(p<0.05)$ and felt more socially lonely $(p<0.05)$ than their counterparts without infection. In the UK, participants with infection were more worried about their family members $(p<0.01)$ than their counterparts. In the USA, no group differences occurred on any of the employed variables. In Australia, the group with infection was too small for meaningful comparison against their counterparts.

Table 2. Worry, loneliness, and mental health among participants with and without COVID-19 infection.

\begin{tabular}{|c|c|c|c|c|c|}
\hline Variables & Without Infection & With Infection & Difference & Effect Size & \\
\hline Worry & $M(S D)$ & $M(S D)$ & $M(S D)$ & Cohen's $d$ & $p$ \\
\hline Worry about own situation & $2.38(1.03)$ & $2.52(1.11)$ & 0.14 & 0.13 & $<0.05$ \\
\hline Worry about immediate family & $2.75(1.00)$ & $2.99(1.03)$ & 0.25 & 0.24 & $<0.001$ \\
\hline Worry about the future & $2.75(1.11)$ & $2.83(1.06)$ & 0.09 & 0.07 & 0.16 \\
\hline \multicolumn{6}{|l|}{ Loneliness } \\
\hline Emotional loneliness & $6.01(2.69)$ & $6.18(2.65)$ & 0.17 & 0.06 & 0.18 \\
\hline Social loneliness & $3.89(2.98)$ & $4.12(3.19)$ & 0.22 & 0.08 & 0.14 \\
\hline \multicolumn{6}{|l|}{ Mental health } \\
\hline GHQ score & $16.19(6.87)$ & $17.09(7.72)$ & 0.90 & 0.13 & $<0.05$ \\
\hline
\end{tabular}

Note. Statistical tests are independent $t$-tests.

\subsection{Factors Associated with Worrying, Loneliness, and Mental Health}

Among those with infection (self or in the family), higher age was associated with lower emotional loneliness and better mental health. Female gender was associated with poorer mental health. Being employed was associated with less worry about the future and lower emotional loneliness, while living with a spouse or partner was associated with lower emotional and social loneliness (see Table 3).

Table 3. Standardized $\beta$ weights of adjusted associations between sociodemographic factors and worrying, loneliness, and mental health among participants with infection $(n=504)$.

\begin{tabular}{|c|c|c|c|c|c|c|}
\hline $\begin{array}{l}\text { Sociodemographic } \\
\text { Variables }\end{array}$ & Worry Self & Worry Family & Worry Future & $\begin{array}{l}\text { Emotional } \\
\text { Loneliness }\end{array}$ & $\begin{array}{c}\text { Social } \\
\text { Loneliness }\end{array}$ & $\begin{array}{l}\text { Mental } \\
\text { Health }\end{array}$ \\
\hline Age group & 0.02 & 0.08 & -0.05 & $-0.19 * * *$ & -0.06 & $-0.13^{* *}$ \\
\hline Gender & 0.10 & -0.04 & -0.02 & 0.04 & -0.04 & $0.09 *$ \\
\hline Education & -0.08 & -0.09 & -0.08 & -0.07 & -0.07 & -0.07 \\
\hline Employment & -0.09 & -0.03 & $-0.15^{* *}$ & $-0.11 *$ & -0.08 & -0.09 \\
\hline $\begin{array}{c}\text { Living with } \\
\text { spouse/partner }\end{array}$ & -0.06 & -0.11 & -0.07 & $-0.13^{* *}$ & $-0.16^{* *}$ & -0.05 \\
\hline Explained variance & $3.2 \%$ & $2.8 \%$ & $4.0 \%$ * & $7.8 \% * * *$ & $4.4 \% * *$ & $4.5 \% * *$ \\
\hline
\end{tabular}

Note. Table content is standardized $\beta$ weights, indicating the strength of the association between the sociodemographic variables and the dependent variables (worrying, loneliness, and mental health) while adjusting for all included sociodemographic variables. Variable coding: higher age group is higher age; higher gender is female; higher education is having bachelor's degree education or higher; higher employment is having full-time or part-time employment (as opposed to not having employment); living with spouse/partner is 1, while not living with spouse/partner is 0 . Higher scores on worry and loneliness indicate higher levels, whereas higher ratings on mental health indicate poorer mental health. ${ }^{*} p<0.05,{ }^{* *} p<0.01,{ }^{* * *} p<0.001$.

Among those without experience of infection, higher age was associated with more worry about one's own situation, less worry about the future, lower emotional loneliness and better mental health. Compared to men, women experienced more worry in all domains, and they experienced more emotional loneliness, less social loneliness and poorer mental health. Having higher education, having employment and living with a spouse or partner were all associated with less worry in all domains, lower emotional and social loneliness, and better mental health (see Table 4). 
Table 4. Standardized $\beta$ weights of adjusted associations between sociodemographic factors and worrying, loneliness and mental health among participants without infection $(n=3306)$.

\begin{tabular}{|c|c|c|c|c|c|c|}
\hline $\begin{array}{c}\text { Independent } \\
\text { Variables }\end{array}$ & Worry Self & Worry Family & Worry Future & $\begin{array}{c}\text { Emotional } \\
\text { Loneliness }\end{array}$ & $\begin{array}{c}\text { Social } \\
\text { Loneliness }\end{array}$ & $\begin{array}{l}\text { Mental } \\
\text { Health }\end{array}$ \\
\hline Age group & $0.05 *$ & -0.02 & $-0.05^{*}$ & $-0.19^{* * *}$ & -0.00 & $-0.16^{* * *}$ \\
\hline Gender & $0.07^{* *}$ & $0.08^{* * *}$ & $0.08^{* * *}$ & $0.04 *$ & $-0.05^{* *}$ & $0.10^{* * *}$ \\
\hline Education & $-0.10^{* * *}$ & -0.04 & $-0.09^{* * *}$ & $-0.08^{* * *}$ & $-0.06^{* * *}$ & $-0.07^{* * *}$ \\
\hline Employment & $-0.09^{* * *}$ & $-0.05^{*}$ & $-0.16^{* * *}$ & $-0.09 * * *$ & $-0.10^{* * *}$ & $-0.10^{* * *}$ \\
\hline $\begin{array}{l}\text { Living with } \\
\text { spouse/partner }\end{array}$ & $-0.06^{* *}$ & $-0.05^{*}$ & $-0.08^{* * *}$ & $-0.18^{* * *}$ & $-0.17^{* * *}$ & $-0.13^{* * *}$ \\
\hline Explained variance & $3.6 \% * * *$ & $1.4 \% * * *$ & $5.7 \% * * *$ & $10.4 \% * * *$ & $4.9 \% * * *$ & $7.1 \% * * *$ \\
\hline
\end{tabular}

Note. Table content is standardized $\beta$ weights, indicating the strength of the association between the sociodemographic variables and the dependent variables (worry, loneliness, and mental health) while adjusting for all included sociodemographic variables. Variable coding: higher age group is higher age; higher gender is female; higher education is having bachelor's degree education or higher; higher employment is having full-time or part-time employment (as opposed to not having employment). Living with spouse/partner is 1 , while not living with spouse/partner is 0 . Higher scores on worry and loneliness indicate higher levels, whereas higher ratings on mental health indicate poorer mental health. ${ }^{*} p<0.05,{ }^{* *} p<0.01,{ }^{* * *} p<0.001$.

\section{Discussion}

This study aimed to examine the differences in worries, loneliness, and mental health between those with and without experience of COVID-19 infection personally or in the immediate family. Despite significantly higher levels of worry and poorer mental health among those experiencing infection, the effect sizes associated with the results demonstrate that differences between those with and without infection experience were small to negligible. The associations between sociodemographic variables and worry, loneliness, and mental health were similar for the two groups, implying that the psychological impacts of COVID-19 may be extended across the population, even among those without infection.

In comparison to previous studies using the GHQ to measure mental health in general populations [25,26], as expected, this study sample had much poorer mental health. The main finding of this study is that the differences between those with and without infection themselves or in the immediate family were small to negligible. This supports the hypothesis that the COVID-19 situation affects the mental health in the general population, and not just the mental health of those who are most directly affected. This interpretation is also supported by other studies of COVID-19 [9,10], suggesting that the COVID-19 outbreak may have adverse psychological impacts beyond the individual, extending to community and global levels.

While statistically significant differences were found regarding worry about own situation, worry about family, and mental health, effect sizes were generally small. The large number of individuals in our sample contributed toward making even very small effect sizes reach statistical significance. The largest effect was found for worry about family members, where those with infection were more worried than those without infection. It is understandable that those with infection felt worried about other family members potentially contracting the disease or worry about family members who had already been infected. In particular, having older family members or family members with underlying chronic disease (cardiovascular disease, chronic obstructive pulmonary disease, hypertension, diabetes and cerebrovascular disease) may have increased worry, given that these diseases are known and major risk factors for poor outcomes among patients with COVID-19 infection [4]. Given that the larger proportion of those 'with infection' consisted of individuals reporting infection in the immediate family, the explanation emphasizing worry for family members who had contracted the disease seems viable in most cases. Rerunning the analyses for each of the countries, the main pattern from the analysis of the whole sample was retained, despite some minor discrepancies and varied baseline levels of infection rates.

For the group without infection in the immediate family, most associations were weak but statistically significant. This is indicative of a high-powered study to detect even very small effects in the data [28]. However, the associations were in the same direction (or were near zero) in both groups of participants. Focusing on associations with effect sizes above 
0.10 , the study showed that higher age was associated with lower emotional loneliness and better mental health in both groups. This was somewhat a surprise finding because older people are at higher risk from COVID-19, so we may have expected higher levels of worry in this age group. However, our finding is mostly in line with previous studies, in which higher age has been associated with less anxiety [29] and less depression [30], but also with more loneliness [31]. However, the age-loneliness association has been found to vary between countries [32]. Further, the association was specifically concerned with emotional loneliness (e.g., feeling rejected), while the association with social loneliness (e.g., having no one to turn if needed) was near zero. The notion of better mental health among those with higher age warrants further investigation. For example, different types of anxiety disorders have been found to have different prevalence across age groups, with phobias having the highest prevalence among children and adolescents, while panic disorder and PTSD are most prevalent in adulthood, and worry (i.e., generalized anxiety disorder) is most prevalent among older adults [32]. While social distancing may affect people similarly across age groups with regard to their possibility of maintaining social interactions, the emotional consequences of reduced social interaction may be more outspoken among individuals of younger age. Older and younger adults may also use social media for different reasons and with different frequency, which may have psychological impacts. Social distancing policies may also affect older adults differently depending on their retirement and residential status.

We found that women had poorer mental health than men. This is in line with a vast amount of research-women have been found to be more susceptible to most types of mental health problems except alcohol and drug misuse, which is more common among men [33-36]. Thus, it appears the COVID-19 situation may have perpetuated the gender gap with respect to mental health.

Among those with infection in the family, having higher education was not significantly associated with any of the psychological outcomes. In those without infection, having higher education was weakly associated with most outcomes. Perhaps of most interest, though, there were no significant differences in worry for family members between participants with and without higher education. Thus, while higher education, by increasing knowledge and self-efficacy, may be a resource for reducing worry about one's own situation and worry about the future, one's worry about family members appears not to vary by education level. This finding adds nuance to the general conception that higher levels of education are associated with better health [37]. Our findings imply that education may not buffer against the worries instigated by the possibility of having family members exposed to an ongoing virus pandemic. If having relevant knowledge about the disease and self-efficacy related to implementing measures of prevention are crucial mechanisms in determining health outcomes [38], the results make sense. While the control over one's own behavior and exposure to risk is substantial, the control one can impose on others' behavior and exposure to risk is minimal.

Having employment was associated with less worry about the future and less loneliness. Among those without infection, employment was also associated with better mental health. During the first phase of the COVID-19 outbreak, many businesses were closed and employees were temporarily furloughed [3]. Given the importance of employment for income and social interactions, it is understandable that those who were employed were less worried and less lonely than their counterparts who were unemployed. Although it might be preferable to conceptualize employment as a continuum rather than a categorical measure [39], having employment per se has previously been shown to be related to lower odds of depression [30] and better global health [40] in a general population sample.

The association between living with spouse/partner and reporting less loneliness and better mental health is similarly understandable. The paired relationship is often the basis for experiencing regular social contact and support, which in turn has been found to be a strong predictor of health outcomes [11]. Not only does the continued affection and support from another person counteract potential effects of stress on mental health [13], 
the paired relationship also allows for experiencing the benefits of providing support to the other person [14].

\section{Study Limitations}

The study is limited in several ways. The data were collected using a cross-sectional online survey, therefore assumptions about causal relationships should not be made. We do not know how well the sample is representative of the population of people who used social media in the four respective countries. Our study sample had a higher proportion of female, well-educated and urban participants. However, the age distributions were generally considered well in accordance with general population statistics. On the other hand, response to the general population targeted advertisement in Australia was low, resulting in a large proportion of participants being recruited among followers of the university's social media postings. Thus, among the Australian participants, there was an over-representation of younger participants with university degrees. However, the sampling of participants from four different countries reduces the risk of severe sampling bias [41].

The sample was recruited through advertisements released by the university through social media and by personal postings and shares on social media. Thus, the results may not be generalized beyond those who use social media relatively frequently. The degree of disease outbreak and social distancing policies differed between states within the USA, which warrants deeper investigation. The internal consistency of the emotional loneliness scale was lower than the recommended 0.70 threshold. However, lower internal consistency estimates are common for shorter scales $[42,43]$. This consideration applies to the three-item emotional loneliness scale. Subpopulations, such as older adults and those who have experienced job loss, should be examined in future research.

\section{Conclusions}

Previous studies concerned with responses to the COVID-19 pandemic have frequently targeted specific groups of interest, whereas studies comparing responses between the general population and those most closely affected - by being infected by the virus themselves, or by having family members infected-appear to be missing. Therefore, this study contributes to the literature by examining differences in worries, loneliness, and mental health between individuals infected by COVID-19 or having someone in their family infected, and the rest of the population. The results of the study showed that those in the group exposed to infection reported more worry about self and the immediate family, and worse mental health, but the differences were small. Thus, the specific contribution of this study lies in its demonstration of mental health effects of the COVID-19 situation extending to the wider population - they are not limited to those who have been infected or have experienced someone with infection in the family. This implies that mental health policies during and following the pandemic need to target the general population as a whole, and not only those who have been most directly affected.

Author Contributions: A.Ø.G. developed the questionnaire in collaboration with T.B., J.L., M.S., H.T., D.P. and M.R., A.Ø.G., M.S., M.R. and J.L. collected the data. T.B. analyzed the data and drafted the manuscript. T.B., J.L., M.S., H.T., D.P., M.R. and A.Ø.G. provided scientific input and critical revisions to the different manuscript drafts, approved the final version to be published, and agreed to be accountable for all aspects of this work. All authors have read and agreed to the published version of the manuscript.

Funding: No funding was obtained for this study.

Data Availability Statement: The dataset analyzed for this study will be available from Oslo Metropolitan University on request after the completion of the study. E-mail amyoge@oslomet.no.

Conflicts of Interest: The authors declare no conflict of interest. 


\section{References}

1. Norwegian Institute of Public Health. The Norwegian Institute of Public Health Specifies Advice on Social Distancing. Available online: https: / / www.fhi.no/nyheter/2020/fhi-presiserer-rad-om-sosial-distansering/ (accessed on 7 June 2021).

2. Ministry of Health. Regulation on Interventions to Prevent the Spread of Contagious Disease etc. by the Corona Outbreak; Ministry of Health: Oslo, Norway, 2020.

3. Blustein, D.L.; Duffy, R.; Ferreira, J.A.; Cohen-Scali, V.; Cinamon, R.G.; Allan, B.A. Unemployment in the time of COVID-19: A research agenda. J. Voc. Behav. 2020, 103436. [CrossRef]

4. Wang, B.; Li, R.; Lu, Z.; Huang, Y. Does comorbidity increase the risk of patients with COVID-19: Evidence from meta-analysis. Aging 2020, 12, 6049-6057. [CrossRef]

5. Jeong, H.; Yim, H.W.; Song, Y.J.; Ki, M.; Min, J.A.; Cho, J.; Chae, J.H. Mental health status of people isolated due to Middle East Respiratory Syndrome. Epidemiol. Health 2016, 38, e2016048. [CrossRef]

6. Hawryluck, L.; Gold, W.L.; Robinson, S.; Pogorski, S.; Galea, S.; Styra, R. SARS control and psychological effects of quarantine, Toronto, Canada. Emerg. Infect. Dis. 2004, 10, 1206-1212. [CrossRef]

7. Lett, H.S.; Blumenthal, J.A.; Babyak, M.A.; Strauman, T.J.; Robins, C.; Sherwood, A. Social support and coronary heart disease: Epidemiologic evidence and implications for treatment. Psychosom. Med. 2005, 67, 869-878. [CrossRef]

8. Wang, C.; Pan, R.; Wan, X.; Tan, Y.; Xu, L.; Ho, C.S.; Ho, R.C. Immediate psychological responses and associated factors during the initial stage of the 2019 Coronavirus disease (COVID-19) epidemic among the general population in China. Int. J. Environ. Res. Public Health 2020, 17, 1729. [CrossRef]

9. Mukhtar, S. Psychological health during the coronavirus disease 2019 pandemic outbreak. Int. J. Soc. Psychiatry 2020, 20764020925835. [CrossRef]

10. Torales, J.; O'Higgins, M.; Castaldelli-Maia, J.M.; Ventriglio, A. The outbreak of COVID-19 coronavirus and its impact on global mental health. Int. J. Soc. Psychiatry 2020. [CrossRef]

11. Reblin, M.; Uchino, B.N. Social and emotional support and its implication for health. Curr. Opin. Psychiatry 2008, 21, 201-205. [CrossRef]

12. Moser, D.K.; Robinson, S.; Biddle, M.J.; Pelter, M.M.; Nesbitt, T.S.; Southard, J.; Cooper, L.; Dracup, K. Health literacy predicts morbidity and mortality in rural patients with heart failure. J. Card Fail. 2015, 21, 612-618. [CrossRef]

13. Berkman, L.F.; Glass, T.; Brissette, I.; Seeman, T.E. From social integration to health: Durkheim in the new millennium. Soc. Sci. Med. 2000, 51, 843-857. [CrossRef]

14. Takizawa, T.; Kondo, T.; Sakihara, S.; Ariizumi, M.; Watanabe, N.; Oyama, H. Stress buffering effects of social support on depressive symptoms in middle age: Reciprocity and community mental health. Psychiatry Clin. Neurosci. 2006, 60, 652-661. [CrossRef] [PubMed]

15. Hysing, M.; Elgen, I.; Gillberg, C.; Lie, S.A.; Lundervold, A.J. Chronic physical illness and mental health in children. Results from a large-scale population study. J. Child. Psychol. Psychiatry 2007, 48, 785-792. [CrossRef] [PubMed]

16. Wang, S.; Li, B.; Ungvari, G.S.; Ng, C.H.; Chiu, H.F.K.; Kou, C.; Liu, Y.; Tao, Y.; Wu, Y.; Fu, Y.; et al. Poor mental health status and its associations with demographic characteristics and chronic diseases in Chinese elderly. Soc. Psychiatry Psychiatr. Epidemiol. 2016, 51, 1449-1455. [CrossRef] [PubMed]

17. Gao, J.; Zheng, P.; Jia, Y.; Chen, H.; Mao, Y.; Chen, S.; Wang, Y.; Fu, H.; Dai, J. Mental health problems and social media exposure during COVID-19 outbreak. PLoS ONE 2020, 15, e0231924. [CrossRef]

18. de Jong Gierveld, J.; van Tilburg, T. A 6-item scale for overall, emotional, and social loneliness. Confirmatory tests on survey data. Res. Aging 2006, 28, 582-598. [CrossRef]

19. Bonsaksen, T.; Opseth, T.M.; Misund, A.; Geirdal, A.Ø.; Fekete, O.R.; Nordli, H. The de Jong Gierveld Loneliness Scale used with Norwegian clubhouse members: Psychometric properties and associated factors. Int. J. Psychosoc. Rehabil. 2018, 22, 88-100.

20. Goldberg, D.P.; Gater, R.; Sartorius, N.; Ustun, T.B.; Piccinelli, M.; Gureje, O.; Rutter, C. The validity of two versions of the GHQ in the WHO study of mental illness in general health care. Psychol. Med. 1997, 27, 191-197. [CrossRef]

21. Goodwin, L.; Ben-Zion, I.; Fear, N.T.; Hotopf, M.; Stansfeld, S.A.; Wessely, S. Are reports of psychological stress higher in occupational studies? A systematic review across occupational and population based studies. PLoS ONE 2013, 8, e78693. [CrossRef]

22. Adlaf, E.M.; Gliksman, L.; Demers, A.; Newton-Taylor, B. The prevalence of elevated psychological distress among Canadian undergraduates: Findings from the 1998 Canadian Campus Survey. J. Am. Coll. Health 2001, 50, 67-72. [CrossRef]

23. Firth, J. Levels and sources of stress in medical students. BMJ 1986, 292, 1177-1180. [CrossRef]

24. Gorter, R.; Freeman, R.; Hammen, S.; Murtomaa, H.; Blinkhorn, A.; Humphris, G. Psychological stress and health in undergraduate dental students: Fifth year outcomes compared with first year baseline results from five European dental schools. Eur. J. Dent. Educ. 2008, 12, 61-68. [CrossRef] [PubMed]

25. Aalto, A.-M.; Elovainio, M.; Kivimäki, M.; Uutela, A.; Pirkola, S. The Beck Depression Inventory and General Health Questionnaire as measures of depression in the general population: A validation study using the Composite International Diagnostic Interview as the gold standard. Psychiatry Res. 2012, 197, 163-171. [CrossRef]

26. Pevalin, D.J. Multiple applications of the GHQ-12 in a general population sample: An investigation of long-term retest effects. Soc. Psychiatry Psychiatr. Epidemiol. 2000, 35, 508-512. [CrossRef] 
27. Fagerland, M.W. T-tests, non-parametric tests, and large studies-A paradox of statistical practice? BMC Med. Res. Methodol. 2012, 12, 78. [CrossRef]

28. Field, A. Discovering Statistics Using SPSS, 4th ed.; Sage Publications: London, UK, 2013.

29. Bonsaksen, T.; Heir, T.; Ekeberg, Ø.; Grimholt, T.K.; Lerdal, A.; Skogstad, L.; Schou-Bredal, I. Self-evaluated anxiety in the Norwegian population: Prevalence and associated factors. Arch. Public Health 2019, 77. [CrossRef]

30. Bonsaksen, T.; Grimholt, T.K.; Skogstad, L.; Lerdal, A.; Ekeberg, Ø.; Heir, T.; Schou-Bredal, I. Self-diagnosed depression in the Norwegian general population-Associations with neuroticism, extraversion, optimism, and general self-efficacy. BMC Public Health 2018, 18, 1076. [CrossRef]

31. Yang, K.; Victor, C. Age and loneliness in 25 European nations. Ageing Soc. 2011, 31, 1368-1388. [CrossRef]

32. Lenze, E.J.; Wetherell, J.L. A lifespan view of anxiety disorders. Dialogues Clin. Neurosci. 2011, 13, 381-399.

33. Kringlen, E.; Torgersen, S.; Cramer, V. A Norwegian psychiatric epidemiological study. Am. J. Psychiatry 2001, 158, 1091-1098. [CrossRef]

34. Kringlen, E.; Torgersen, S.; Cramer, V. Mental illness in a rural area: A Norwegian psychiatric epidemiological study. Soc. Psychiatry Psychiatr. Epidemiol. 2006, 41, 713-719. [CrossRef]

35. Alonso, J.; Angermeyer, M.C.; Bernert, S.; Bruffaerts, R.; Brugha, T.S.; Bryson, H.; de Girolamo, G.; Graaf, R.; Demyttenaere, K.; Gasquet, I.; et al. Prevalence of mental disorders in Europe: Results from the European Study of the Epidemiology of Mental Disorders (ESEMeD) project. Acta Psychiatr. Scand. Suppl. 2004, 21-27. [CrossRef]

36. McHugh, R.K.; Votaw, V.R.; Sugarman, D.E.; Greenfield, S.F. Sex and gender differences in substance use disorders. Clin. Psychol. Rev. 2018, 66, 12-23. [CrossRef]

37. Fletcher, J.M.; Frisvold, D.E. Higher education and health investments: Does more schooling affect preventive health care use? J. Hum. Cap. 2009, 3, 144-176. [CrossRef]

38. Adams, R.J. Improving health outcomes with better patient understanding and education. Risk Manag. Healthc. Policy 2010, 3, 61-72. [CrossRef]

39. Dooley, D. Unemployment, underemployment, and mental health: Conceptualizing employment status as a continuum. Am. J. Community Psychol. 2003, 32, 9-20. [CrossRef]

40. Bonsaksen, T.; Ekeberg, Ø.; Skogstad, L.; Heir, T.; Grimholt, T.K.; Lerdal, A.; Schou-Bredal, I. Self-rated global health in the Norwegian general population. Health Qual. Life Outcomes 2019, 17, 188. [CrossRef]

41. Brouwer, R.; Veldhuisen, D.; Boer, R. Surviving the first COVID-19 wave and learning lessons for the second. Eur. J. Heart Fail. 2020, 22, 975-977. [CrossRef]

42. Streiner, D.L.; Norman, G.R. Health Measurement Scales-A Practical Guide to Their Development and Use, 4th ed.; Oxford University Press: Oxford, UK, 2008.

43. Ponterotto, J.G.; Ruckdeschel, D. An overview of coefficient alpha and a reliability matrix for estimating adequacy of internal consistency coefficients with psychological research measures. Percept. Mot. Ski. 2007, 105, 997-1014. [CrossRef] 